\title{
REVIEW
}

Open Access

\section{The efficacy and safety of combined immune checkpoint inhibitors (nivolumab plus ipilimumab): a systematic review and meta-analysis}

Jingjie Chen ${ }^{1,2,3 \dagger}$, Shengnan $\mathrm{Li}^{4+}$, Qigu Yao ${ }^{5}$, Nannan Du', Xiaojun Fu', Yuanmei Lou' ${ }^{6}$, Mengru Wang ${ }^{7}$, Feiyan Mao ${ }^{1}$, Danyi Mao ${ }^{8}$, Parikshit Asutosh Khadaroo ${ }^{9}$ and Yingying Tang ${ }^{10^{*}}$

\begin{abstract}
Background: Currently, nivolumab and ipilimumab are the most widely used immune checkpoint inhibitors. We performed a meta-analysis to evaluate the efficacy and treatment-related adverse events (TRAEs) of nivolumab plus ipilimumab therapy in cancer treatment.

Methods: We examined data from PubMed, Web of Science, EBSCO, and Cochrane Library. Eleven articles fulfilled our criteria, which we divided into 3 groups: nivolumab plus ipilimumab versus nivolumab (the dose used for monotherapy is $3 \mathrm{mg} / \mathrm{kg}$ ), nivolumab plus ipilimumab versus ipilimumab (the dose used for monotherapy is $3 \mathrm{mg} /$ $\mathrm{kg}$ ), and nivolumab $1 \mathrm{mg} / \mathrm{kg}$ plus ipilimumab $3 \mathrm{mg} / \mathrm{kg}$ (N1/3) versus nivolumab $3 \mathrm{mg} / \mathrm{kg}$ plus ipilimumab $1 \mathrm{mg} / \mathrm{kg}$ (N311). We measured the complete response (CR), partial response (PR), objective response rate (ORR), and TRAEs in any grade and grade 3 or higher.

Results: The overall effect estimate favored the combined immunotherapy group in terms of the ORR (RR: 1.40, $p<$ $0.001)$ and PR (RR: 1.50, $p<0.001$ ) than nivolumab alone. Compared with ipilimumab alone, the combined immunotherapy group had better CR (RR: 4.89, $p<0.001$ ), PR (RR: 2.75, $p<0.001$ ), and ORR (RR: 3.31, $p<0.001$ ). Finally, N1I3 showed better PR (RR: 1.35, $p=0.006$ ) and ORR (RR: 1.21, $p=0.03$ ) than N311. The incidence of any TRAEs was similar between both groups (RR: 1.05, $p=0.06$ ). However, the incidence of serious adverse events (grade 3 or higher) was lower in group N311 than group N1I3 (RR: 1.51, $p<0.001$ ).

Conclusion: This meta-analysis showed that the curative effect of nivolumab plus ipilimumab was better than that of nivolumab or ipilimumab monotherapy. In the combined immunotherapy group, N113 was more effective than N311. Although the side effects were slightly increased in N1/3 group, overall safety was acceptable.
\end{abstract}

Keywords: Nivolumab, Ipilimumab, Tumor response, Adverse events, Meta-analysis

\footnotetext{
* Correspondence: ningbohwamei@163.com

${ }^{\dagger}$ Jingjie Chen and Shengnan Li contributed equally to this work.

${ }^{10}$ Department of Radiotherapy and Chemotherapy, HwaMei Hospital,

University of Chinese Academy of Sciences, Northwest Street 41, Haishu

District, Ningbo 315010, Zhejiang, China

Full list of author information is available at the end of the article
}

(C) The Author(s). 2020 Open Access This article is licensed under a Creative Commons Attribution 4.0 International License, which permits use, sharing, adaptation, distribution and reproduction in any medium or format, as long as you give appropriate credit to the original author(s) and the source, provide a link to the Creative Commons licence, and indicate if changes were made. The images or other third party material in this article are included in the article's Creative Commons licence, unless indicated otherwise in a credit line to the material. If material is not included in the article's Creative Commons licence and your intended use is not permitted by statutory regulation or exceeds the permitted use, you will need to obtain permission directly from the copyright holder. To view a copy of this licence, visit http://creativecommons.org/licenses/by/4.0/ The Creative Commons Public Domain Dedication waiver (http://creativecommons.org/publicdomain/zero/1.0/) applies to the data made available in this article, unless otherwise stated in a credit line to the data. 


\section{Background}

Cytotoxic T lymphocyte-associated protein 4 (CTLA-4) is a receptor on the surface of activated $T$ cells [1]. It mainly acts by binding B7 ligand on antigen-presenting cells (APCs). The protein competes with the cluster of differentiation 28 (CD28) receptor for B7 ligand. During $\mathrm{T}$ cell activation, CD28 receptors on T cells bind to B7 ligand on antigen-presenting cells (APCs) and provide the essential second activation signal for $\mathrm{T}$ cells [2-4]. Programmed cell death protein 1 (PD-1) is a cell-surface receptor commonly found in $\mathrm{T}$ cells, B cells, and NK cells [5]. By inhibiting the phosphatidylinositol 3-kinase (PI3K) pathway, PD-1 signaling inhibits the activation of the cell survival factor Bcl-xL and the expression of transcription factors such as GATA-3, T-bet, and Eomes [6], that regulate $\mathrm{T}$ cell functions. The CTLA-4 and PD-1, two common immune checkpoint inhibitors (ICIs) on activated $\mathrm{T}$ cells, are the most reliable targets for cancer treatment. Up to now, seven drugs targeting CTLA-4/ PD-1 have been approved for treatment of different types of cancer, including melanomas [7], lung [8], breast [9], cervical [10], and liver cancer [11].

In clinical studies, CTLA-4 and PD- 1 monotherapy inhibitors have shown impressive, lasting effects that have significantly prolong the survival of responsive patients $[12,13]$. For example, colorectal cancer $(\mathrm{CRC})$ is the third most common cancer in the USA. Despite advances in chemotherapy, survival rates in patients with metastatic CRC remains low. However, CRC patients treated with immune checkpoint inhibitors showed better response [14]. Hepatectomy is an important method to treat liver cancer, but up to $70 \%$ of patients may have a recurrence of liver diseases within 5 years, even after receiving treatment for hepatocellular carcinoma (HCC) at an early stage. However, immunotherapy has recently been shown to be effective against HCC, marking a milestone in the history of this intractable disease [15]. The efficacy of immune-monotherapy is limited by low response rates, with only a small proportion of patients responding to treatment [13]. Rotte et al. [16] reported that drugs when administered as monotherapy had a manageable safety profile, but more than $50 \%$ of patients failed to respond to treatment. While the results of monotherapy treatments are not satisfactory, there is increasing emphasis on combination treatments in an effort to increase response rates to treatment. Combination of CTLA-4 and PD-1 blockers was then evaluated to increase the response rates in patients, and the combination of nivolumab plus ipilimumab showed significantly enhance efficacy in metastatic melanoma patients. The combination group has demonstrated numerically higher response rates and improved long-term clinical benefit relative to anti-PD-1/PD-L1 or antiCTLA-4 monotherapy [14]. Durable responses and encouraging survival have been demonstrated with immune checkpoint inhibitors in small-cell lung cancer (SCLC), and the results showed that nivolumab plus ipilimumab appeared to provide a greater clinical benefit than nivolumab monotherapy in the high tumor mutational burden tertile [17]. Combination of immunotherapies is one of the most promising new methods. Presently, nivolumab and ipilimumab are the most widely used immune checkpoint inhibitors against cancer [18]. This meta-analysis aimed at investigating the role of nivolumab plus ipilimumab therapy in cancer treatment.

\section{Materials and methods}

\section{Search and selection}

We did a meta-analysis of relevant articles, published before by June 2019. We searched through four electronic databases; PubMed, Web of Science, EBSCO, and Cochrane Library for data with relevant clinical trials, based on these keywords: (nivolumab or PD-1 or programmed death 1) and (ipilimumab or CTLA-4 or cytotoxic T-lymphocyte-associated protein 4).

\section{Inclusion and exclusion criteria}

Studies in the selected articles were to meet four criteria: (1) participants: solid tumor patients receiving combined ICIs (nivolumab plus ipilimumab); (2) intervention: combined ICIs including nivolumab and ipilimumab simultaneously; (3) comparisons: nivolumab or ipilimumab alone; (4) outcomes: objective response rate (ORR), partial response rate $(\mathrm{PR})$, complete response $(\mathrm{CR})$, and treatment-related adverse events (TRAEs), otherwise they were excluded. And these studies would be excluded if they were (1) not included in the combination of nivolumab and ipilimumab; (2) non-prospective clinical trials; (3) included other treatments; (4) single-arm study; (5) not relevant outcome.

\section{Data extraction}

We focused on trial phases, tumor types, the number and characteristics of participants, the anti-tumor agents, dosage, and frequency of drug administration with a keen interest on the prognoses, specifically the ORR, CR, and PR. The curative effects were assessed using the Response Evaluation Criteria in Solid Tumors (RECIST). Incidence of TRAEs, including any grade and grade 3 or higher was also evaluated, based on the National Cancer Institute's Common Terminology Criteria for Adverse Events (CTCAE).

\section{Quality assessment}

We evaluated the quality of data in the relevant articles using the Cochrane Collaboration tool based on the following domains: allocation concealment, masking of 
outcome assessors, blinding of participants, incomplete follow-up, and selective outcome reporting.

\section{Statistical analysis}

Statistical analyses were done by the Review Manager software (RevMan5.3), at 95\% confidence intervals (CI). Subgroup analysis was done based on the intervention: nivolumab plus ipilimumab versus nivolumab alone, nivolumab plus ipilimumab versus ipilimumab alone, nivolumab $1 \mathrm{mg} / \mathrm{kg}$ plus ipilimumab $3 \mathrm{mg} / \mathrm{kg}$ (N1I3) versus nivolumab $3 \mathrm{mg} / \mathrm{kg}$ plus ipilimumab $1 \mathrm{mg} / \mathrm{kg}$ (N3I1). All analyses (ORR, CR, PR, and all-grade and serious grade TRAEs) were performed based on the fixed/random-effect. For meta-analysis, we used risk ratios (RR) to compare with dichotomous variables. Heterogeneity in the meta-analysis results was done by the $I$-square $\left(I^{2}\right)$ test. Statistically significant was measured at $P$ values of 0.05 .

\section{Results}

\section{Literature search}

We identified 4361 studies in the literature search. It then removed 2133 duplicates and further 2228 after careful evaluation. Although 56 articles met the inclusion criteria, 45 were removed besides qualifying for meta-analysis for a number of reasons. Among them, 16 articles were excluded because they did not include the combination of nivolumab and ipilimumab; 11 articles were non-prospective clinical trials; 7 articles were excluded because they included other treatments; 6 articles were single-arm studies; 5 articles were excluded because of irrelevant outcome. In the end, 11 articles [1929] were qualified for the meta-analysis. The specific reasons are shown in Supplementary Figure 1.

\section{Study characteristics}

The characteristics of each study are shown in Supplementary Table 1 . The 11 clinical trials included 2484 patients. Of these 879 received nivolumab $1 \mathrm{mg} / \mathrm{kg}$ plus ipilimumab $3 \mathrm{mg} / \mathrm{kg}$ (N1I3), 560 received nivolumab 3 $\mathrm{mg} / \mathrm{kg}$ plus ipilimumab $1 \mathrm{mg} / \mathrm{kg}$ (N3I1), when combined, nivolumab is generally used $3 \mathrm{mg} / \mathrm{kg}$, ipilimumab used 1 $\mathrm{mg} / \mathrm{kg}$ or nivolumab used $1 \mathrm{mg} / \mathrm{kg}$, ipilimumab used 3 $\mathrm{mg} / \mathrm{kg}$. Six hundred eighty-eight received nivolumab at a recommended dose of $3 \mathrm{mg} / \mathrm{kg}$ monotherapy, while 357 were put on ipilimumab at a recommended dose of 3 $\mathrm{mg} / \mathrm{kg}$ monotherapy. The selected studies had varied cases, from melanoma, metastatic urothelial carcinoma, small cell lung cancer (SCLC), esophagogastric cancer (EGC), malignant pleural mesothelioma (MPM), renal cell carcinoma (RCC), sarcoma, glioblastoma. There were two phase I clinical trials, two phase I-II clinical trials, four phase II clinical trials, two phase III clinical trials, and one phase III-IV clinical trials.

\section{Nivolumab plus ipilimumab versus ipilimumab alone}

Compared with ipilimumab alone, nivolumab plus ipilimumab synergy caused a greater effect under CR (RR: 4.89, 95\% CI: $2.91-8.23, p<0.001$ ), PR (RR: $2.75,95 \%$ CI: $2.05-3.69, p<0.001$ ), and ORR (RR: $3.31,95 \% \mathrm{CI}$ :

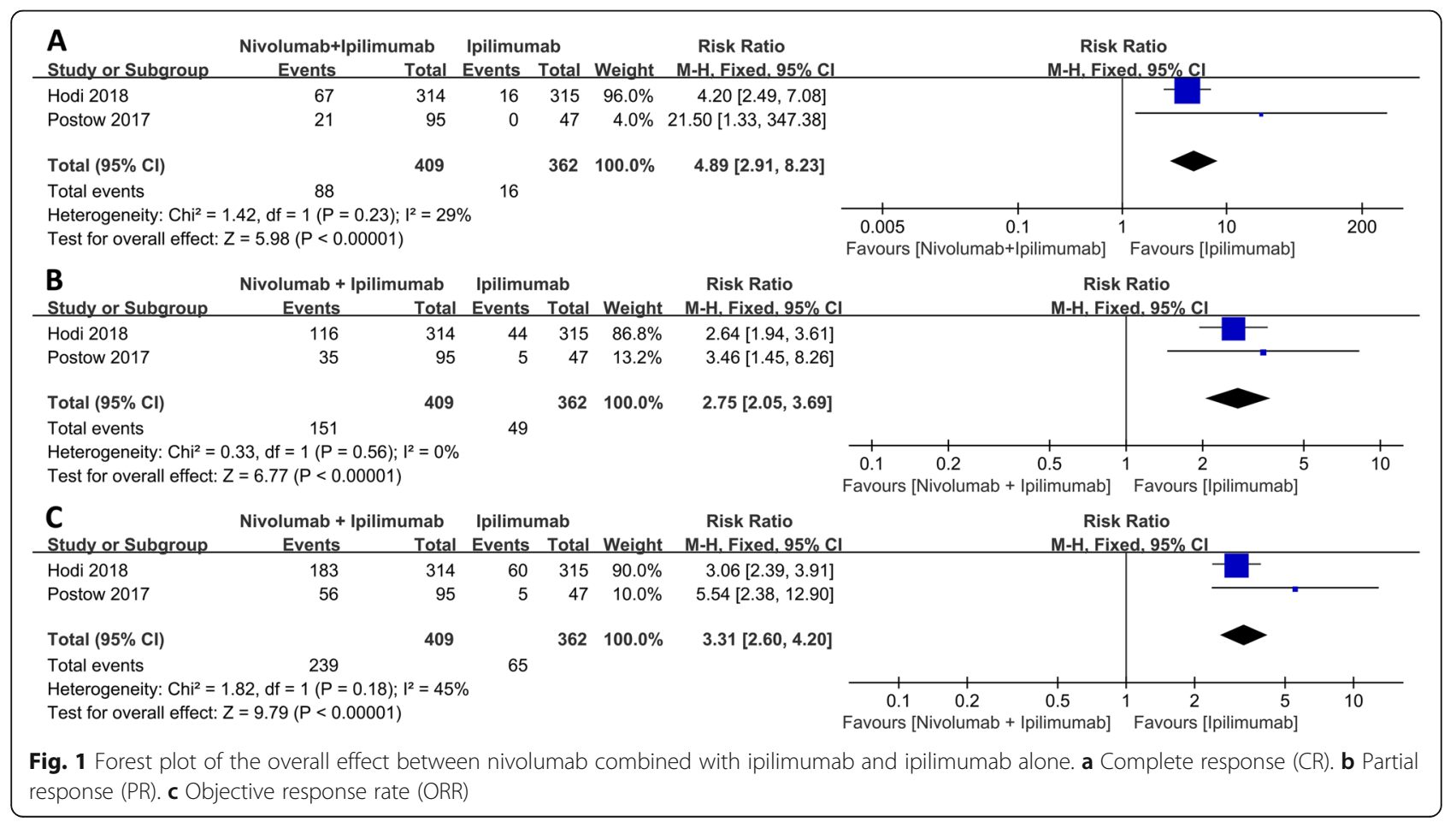


2.60-4.20, $p<0.001$ ) as shown in Fig. 1. Although the incidence of any TRAEs was similar between the two groups (RR: $1.05, p=0.44$ ), ipilimumab monotherapy resulted in less serious cases, (grade 3 or higher) than nivolumab plus ipilimumab group (RR: 2.16, 95\% CI: 1.78-2.61, $p<0.001)$ as shown in Fig. 2.

\section{Nivolumab plus ipilimumab versus nivolumab alone}

Overall, nivolumab plus ipilimumab group showed better ORR (RR: 1.40, 95\% CI: $1.22-1.61, p<0.001)$ and PR (RR: $1.50,95 \%$ CI: $1.23-1.83, p<0.001$ ) than nivolumab alone; however, there was no statistically significant difference in the CR (RR: $1.13, p=0.39$ ) between the two as shown in Fig. 3.

In terms of adverse effects, the incidence of any TRAEs and serious TRAEs were elevated in nivolumab monotherapy than in nivolumab plus ipilimumab group (RR: $1.10,95 \%$ CI: $1.00-1.21, p=0.04$; RR: 2.10 , 95\% CI: $1.57-2.81, p<0.001$, respectively) as shown in Fig. 4 .

\section{N1I3 versus N3I1}

Since the combined therapeutic effect was better than that of monotherapies, subgroup analysis of the combination therapy was further investigated. The N1I3 group showed better PR (RR: 1.35, 95\% CI: 1.09-1.68, $p=$ 0.006 ) and ORR (RR: $1.21,95 \%$ CI: $1.02-1.44, p=0.03$ ), while there was no significant difference in CR (RR: 0.83, $p=0.40$ ) between the two subgroups as shown in Fig. 5. There was no difference in TRAEs between the two groups as well (RR: $1.05, p=0.06$ ); however, N113 produced more serious adverse events (grade 3 or higher) than group N3I1 (RR: 1.51, 95\% CI: 1.27-1.78, $p<$ 0.001) as shown in Fig. 6).

Specific adverse treatment events by subgroup were also analyzed. Incidences of any grade adverse events were more elevated in group N1I3. These include increased alanine aminotransferase (ALT) (RR: 1.48, $p=$
0.02), increased aspartate aminotransferase (AST) (RR: 1.68, $p=0.004)$, diarrhea (RR: 1.47, $p=0.005)$, hypothyroidism (RR: $1.40, p=0.04$ ), and vomiting (RR: 1.77, $p=0.02$ ). As shown in Table 1 , adverse reactions, such as high ALT (RR: 2.25, $p=0.006$ ) and diarrhea (RR: $2.90, p<0.001$ ), of grade 3 or above, were also high in group N1I3 than in group N3I1.

\section{Discussion}

Immunotherapy plays an important role in controlling tumors. Combination immunotherapy is based on the use of more than one immunotherapy. It can intervene and regulate multiple processes of the immune response through [30], chemoradiotherapy [31-33], and targeted therapy $[34,35]$ by promoting anti-tumor immune response reduce the risk of drug resistance. The combination of immunotherapies is one of the most promising approaches being studied [36]. In particular, the combination of anti-PD-1 (nivolumab) and anti-CTLA-4 (ipilimumab) has shown positive results in tumor treatment and significant enhancement in patients with metastatic melanoma [37], advanced RCC [38], and metastatic CRC $[16,39]$. Cope et al. reported that recurrent SCLC showed better response to nivolumab plus ipilimumab compared to the current chemotherapy, interpreted as long-term survival benefits [40]. Ready et al. reported that the combination of nivolumab and low-dose ipilimumab was effective and tolerable as a first-line treatment of advanced/metastatic non-small cell lung cancer (NSCLC) [41]. Based on conditional survival analysis of first-line treatment, Shao [42] showed that patients with advanced RCC put on nivolumab plus ipilimumab therapy had a high survival rate compared with sunitinib.

Recently, the combination therapy of ipilimumab and anti-PD-1 antibody showed promising clinical benefit in some malignant tumors [43], advanced melanoma [44], RCC and other tumors [45]. Both combination therapy

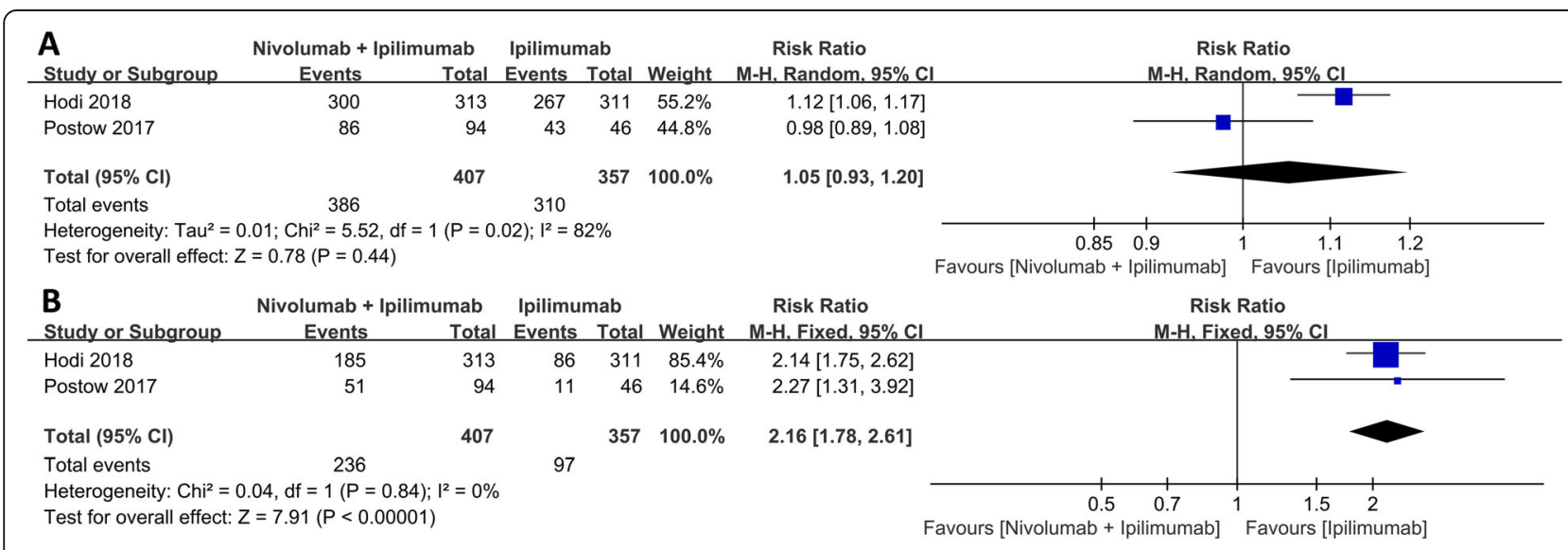

Fig. 2 Forest plot of the adverse events between nivolumab combined with ipilimumab and ipilimumab alone. a Any grade TRAEs. b Grade 3 or higher TRAES 


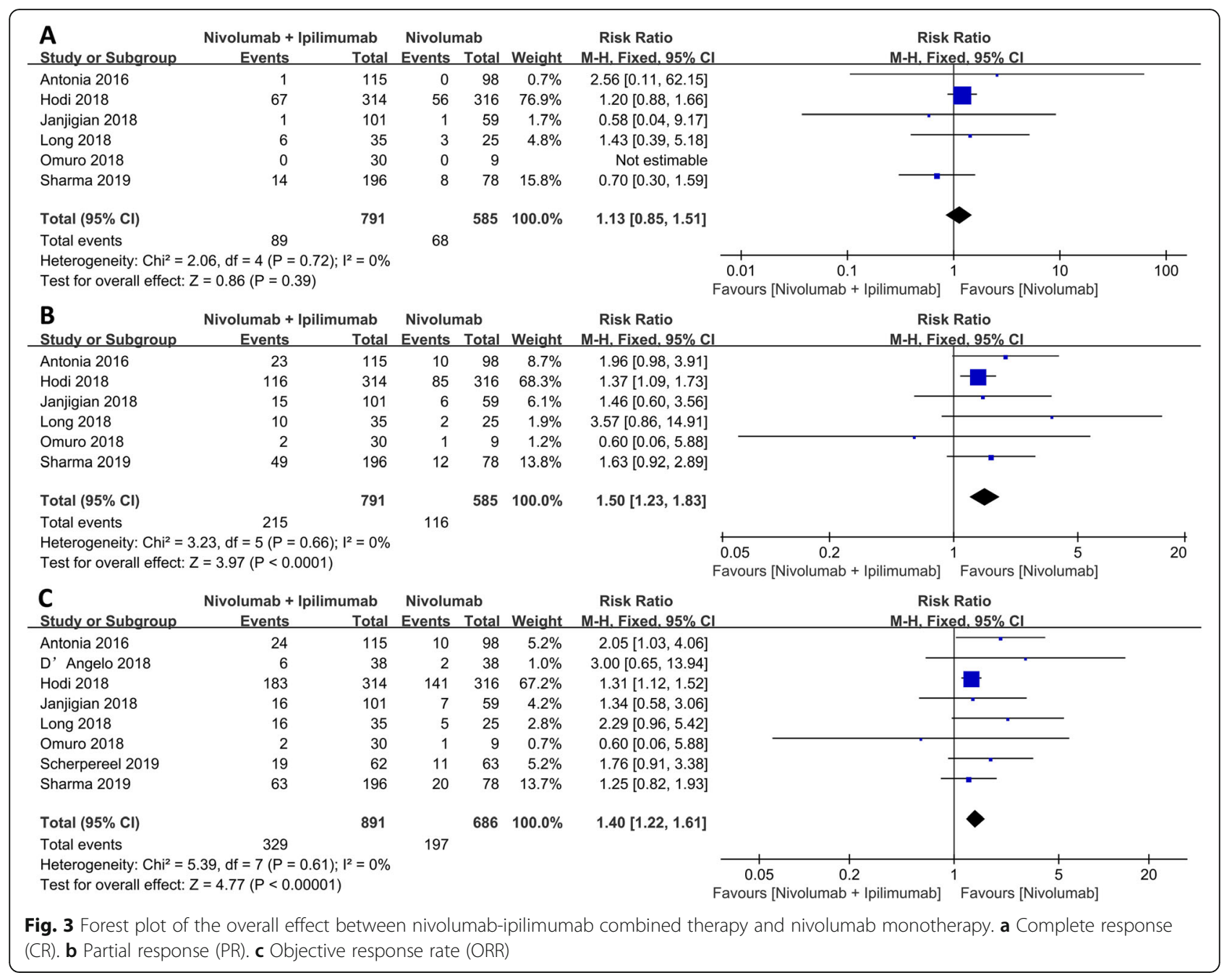

and nivolumab or ipilimumab monotherapy showed improved ORR, CR, and PR [38, 42, 46, 47]. The present systematic review showed that nivolumab plus ipilimumab group had significantly higher CR, PR, and ORR than ipilimumab monotherapy. CR with nivoluma plus ipilimumab therapy was 4.89 times higher than ipilimumab monotherapy, while the PR and ORR were 2.75 and 3.31 times than ipilimumab monotherapy, respectively. These findings show that the combination therapy was more effective than ipilimumab monotherapy. Elsewhere, Postow et al. [27] reported that the combination of nivolumab plus ipilimumab had a higher ORR and progression-free survival rate compared with ipilimumab monotherapy, in treatment-naive patients with advanced melanoma. Increased response rate and improved progression-free survival were reported in nivolumab plus ipilimumab group when compared with ipilimumab alone in a randomized phase III trial in treatment-naive patients with metastatic melanoma [48]. David et al. [49] compared the quality-adjusted survival rates of nivolumab plus ipilimumab group with ipilimumab monotherapy in untreated advanced melanoma patients. The result showed a combination group resulted in a statistically significant and clinically important improvement in quality-adjusted survival.

Nivolumab is a class of ICIs that PD-1 receptors that activate downstream signaling pathways by inducing FoxP3 expression [50] and promoting Treg (iTreg) cell differentiation [16]. The incidence of $C R$ and $P R$ and ORR in individuals on the combined immunotherapy group was $1.13,1.50$, and 1.40 times respectively, as high than those on nivolumab monotherapy. These findings emphasized the effectiveness of the combination therapy. Antonia et al .[19] reported that the combination of nivolumab plus ipilimumab had a higher prolonged antitumor activity in previously treated patients than nivolumab monotherapy. Preliminary data on metastatic RCC suggests that combination therapy had a higher ORR than nivolumab monotherapy in different trials [21, 51]. Morse [14] reported that a combination therapy (nivolumab with low-dose ipilimumab) had numerically higher response rates and improved long-term clinical benefit 


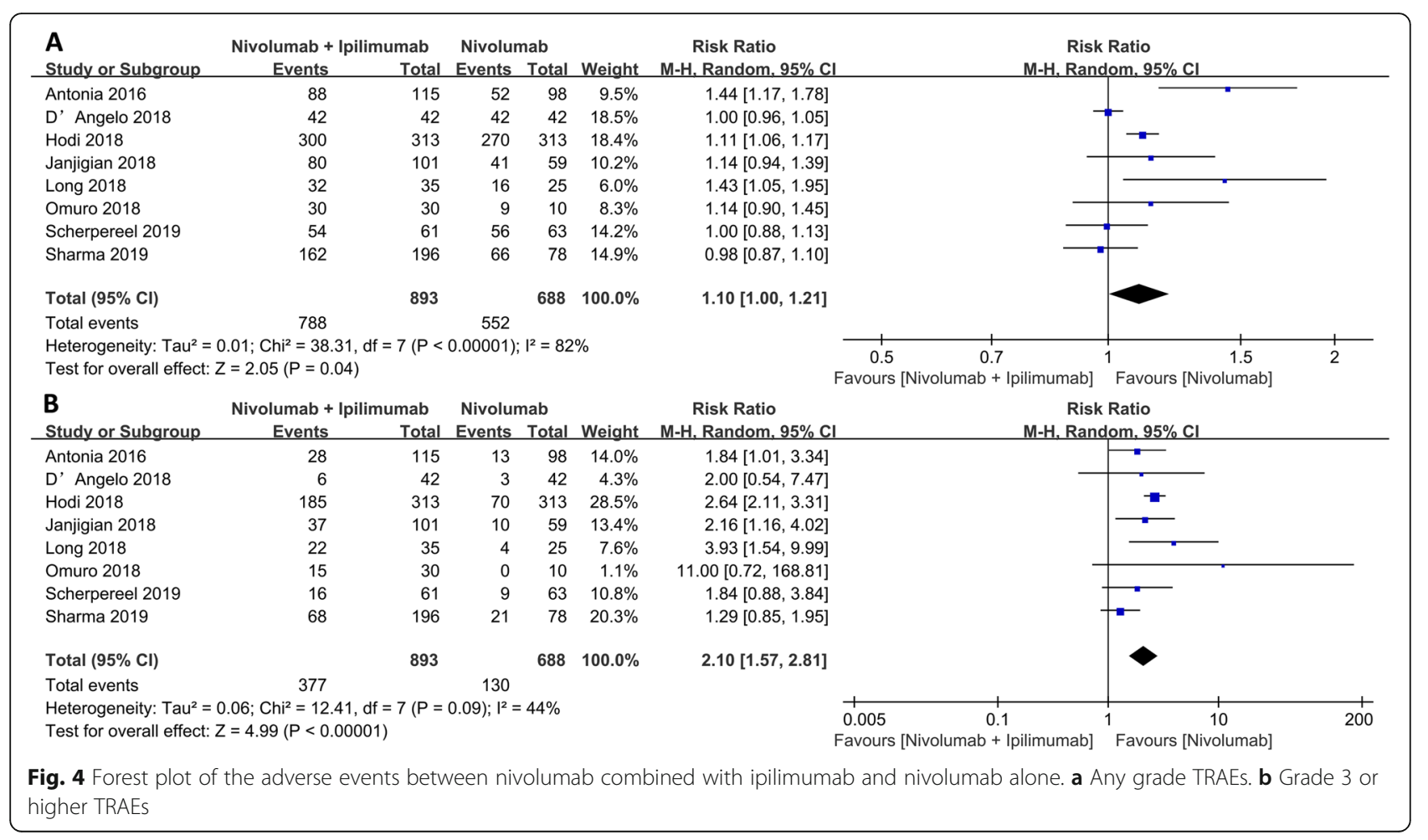

relative to anti-programmed death-1 monotherapy. Principally, CTLA-4 binds B7 ligand (B7-1/CD80 and B7-2/ CD86) on antigen-presenting cells that compete with the CD28 receptor [16]. The CTLA-4 protein and its B7 ligand are mainly expressed on immune cells, suggesting that the CTLA-4 pathway plays a major role in lymph nodes. PD-L1, the PD-1 ligand, is widely expressed, mainly on regulatory peripheral $\mathrm{T}$ cell [52]. Although CTLA-4 and PD-1 antibodies are both checkpoint inhibitors, their action mechanisms are neither the same nor complementary [53]. Therefore, higher anti-tumor activity was seen with a combination therapy than ipilimulab or nivolumab [54].

Based on the above analysis, we can conclude that the combination of nivolumab plus ipilimumab is more effective than nivolumab alone, consistent with previous findings $[19,20]$. Ghiringhelli et al. testified that checkpoint monotherapy inhibitors targeting PD-1 and PD-L1 were not effective in metastatic colorectal cancer patients with microsatellite stable tumors [55]. Neal compared nivolumab plus ipilimumab and nivolumab monotherapy in recurrent SCLC, whereas ORR was higher with nivolumab plus ipilimumab versus nivolumab, toxicities were more common with combination therapy versus nivolumab monotherapy [56]. However, what is different from the above research results is Kreft [57] showed that there was no difference in action and outcome between nivolumab plus ipilimumab group and nivolumab monotherapy in patients with melanoma.
Many combination immunotherapies have been developed, nivolumab plus ipilimumab being the most common [58]. There are two dosages of this combination: nivolumab $1 \mathrm{mg} / \mathrm{kg}$ plus ipilimumab $3 \mathrm{mg} / \mathrm{kg}$ (N1I3) and nivolumab $3 \mathrm{mg} / \mathrm{kg}$ plus ipilimumab $1 \mathrm{mg} / \mathrm{kg}$ (N3I1); however, no studies have been done to determine their differential effectiveness. Although there was no significant difference between N1I3 and N3I1 in CR, N1I3 yielded better resulted in PR and ORR than N3I1, suggesting that the efficacy of N1I3 may be better than that of N3I1. Sharma et al. [29] reported that with longer follow-up, N1I3 showed sustained antitumor activity than N3I1. Glutsch on his part investigated the presence and extent of side effects in advanced melanoma cases on nivolumab plus ipilimumab group. Here, renal toxicity was tolerable, and three doses of nivolumab $(1 \mathrm{mg} /$ $\mathrm{kg})$ in combination with ipilimumab $(3 \mathrm{mg} / \mathrm{kg})$ showed deep partial relief on chest and abdominal CT scans [59]. This result not only supports our findings on N1I3, but emphasized on the potential benefit of combination immunotherapies in the tumor.

We also analyzed all common adverse reactions of every grade. Adverse reactions of grade 3 or higher in the combination group were elevated than those in monotherapy. Nivolumab plus ipilimumab group increased response rate with more side effects than ipilimumab monotherapy. Kreft [57] reported that nivolumab plus ipilimumab group was associated with a higher TRAEs compared with monotherapy, but N1I3 


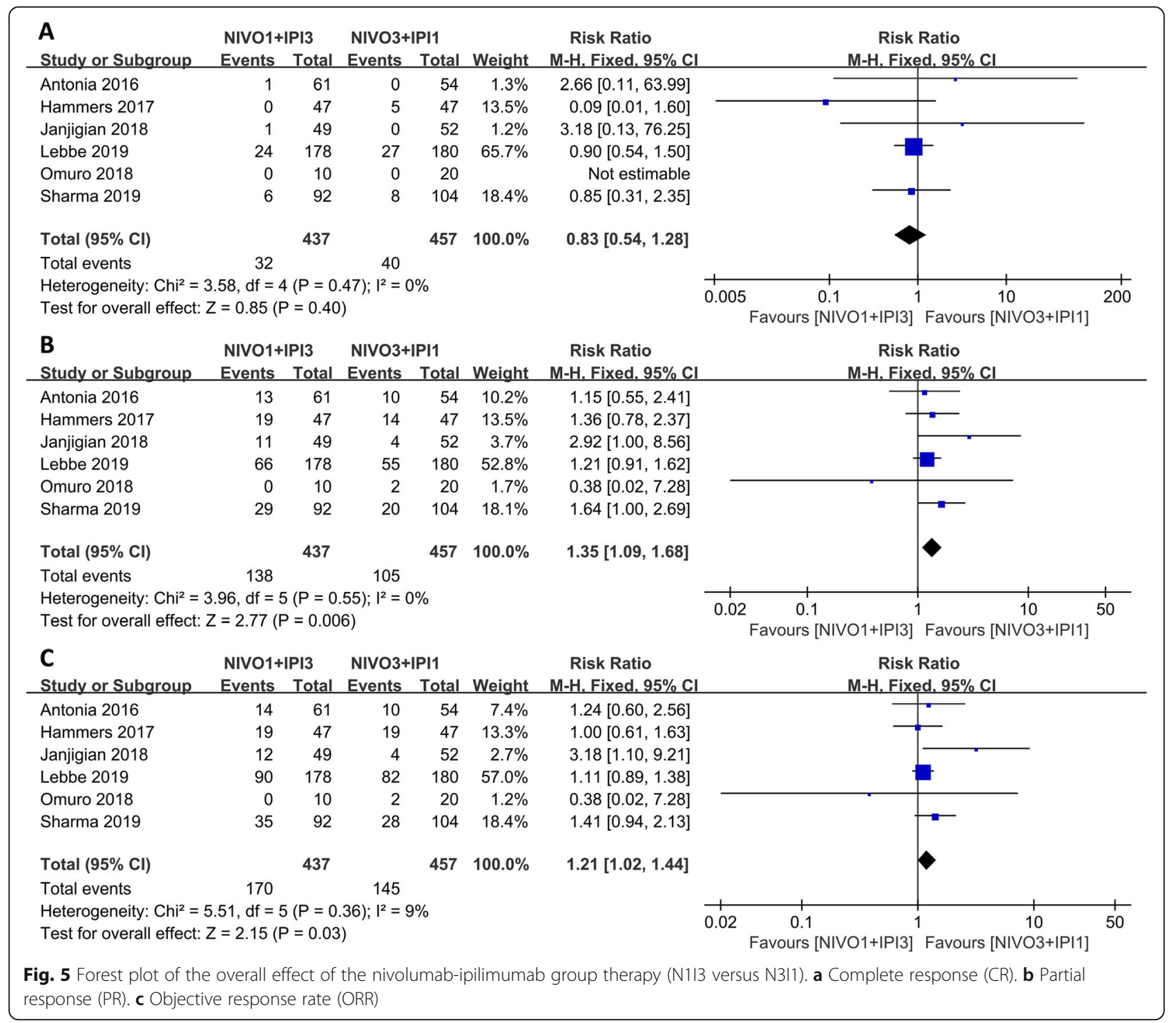

induced elevated grade 3 or higher TRAEs than N3I1, consistent with Antonia et al. [19]. Compared with N3I1, N1I3 improved the treatment benefit and slightly increased the incidence of TRAEs. What we need to make clear is that although N1I3 increased the rate of TRAEs, the overall incidence of TRAEs is controllable, and after symptomatic treatment, most of the conditions can be improved. When comparing with treatment groups, common grade 3 or 4 TRAEs in the nivolumab plus ipilimumab group arose early but resolved within the first 4-6 months of treatment [47]. Most selected treatment-related adverse events occurring within 30 days of the last dose in the nivolumab plus ipilimumab group were low-grade, and the majority resolved and were manageable using established algorithms [60].

The main TRAEs associated with nivolumab use include increased ALT and AST, pruritus, diarrhea, fatigue, nausea, hypothyroidism, decreased appetite, vomiting, and rash. After an extensive systematic review, Bajwa et al. found that the most common adverse effects encountered were colitis (14/139), hepatitis (11/139), adrenocorticotropic hormone insufficiency (12/139), hypothyroidism (7/139), type 1 diabetes (22/139), acute kidney injury (16/139), and myocarditis (10/139). The most common treatment approach was the cessation of the immune checkpoint inhibitor, initiation of steroids and supportive therapy $[61,62]$. Motzer et al. reported that among all patients treated, the most common TRAEs in the grade 3-4 nivolumab plus ipilimumab group were elevated lipase (57 of $547[10 \%])$, elevated amylase (31 [6\%]), and elevated ALT (28 [5\%] )[47]. Reporting of corticosteroid use for ICIs has been effective among various studies. 


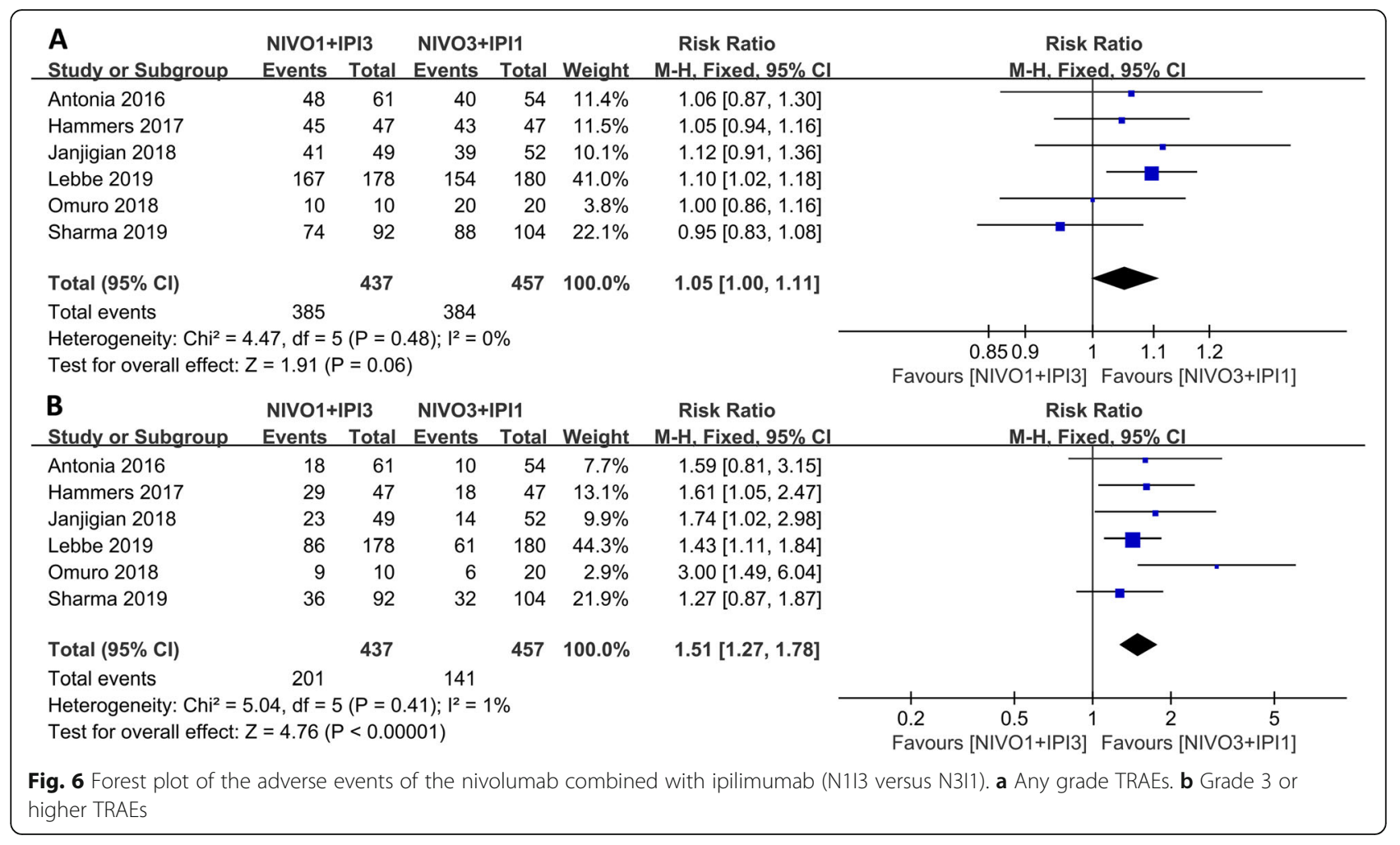

Table 1 Subgroup analysis of the treatment-related adverse events (TRAEs)

\begin{tabular}{|c|c|c|c|c|c|c|c|}
\hline \multirow[t]{2}{*}{$\mathrm{NIVO1}+$ IPI3 vs. NIVO3 + IPI1 } & \multirow{2}{*}{$\begin{array}{l}\text { No. of } \\
\text { studies }\end{array}$} & \multirow[t]{2}{*}{ RR } & \multirow[t]{2}{*}{$95 \% \mathrm{Cl}$} & \multirow[t]{2}{*}{$p$} & \multirow{2}{*}{$\begin{array}{l}\text { Effect } \\
\text { model }\end{array}$} & \multicolumn{2}{|c|}{ Heterogeneity } \\
\hline & & & & & & $\overline{\left(l^{2}\right)}$ & $p$ \\
\hline Any grade increased ALT & 6 & 1.48 & $1.06-2.06$ & 0.02 & Fixed & $33 \%$ & 0.19 \\
\hline Any grade increased AST & 6 & 1.68 & $1.18-2.39$ & 0.004 & Fixed & $41 \%$ & 0.13 \\
\hline Any grade in pruritus & 6 & 1.09 & $0.87-1.37$ & 0.46 & Fixed & $0 \%$ & 0.53 \\
\hline Any grade in diarrhea & 6 & 1.47 & $1.18-1.83$ & 0.005 & Fixed & $23 \%$ & 0.26 \\
\hline Any grade in fatigue & 6 & 1.06 & $0.88-1.29$ & 0.53 & Fixed & $19 \%$ & 0.29 \\
\hline Any grade in nausea & 5 & 1.34 & $0.99-1.81$ & 0.06 & Fixed & $0 \%$ & 0.63 \\
\hline Any grade in hypothyroidism & 5 & 1.40 & $1.01-1.94$ & 0.04 & Fixed & $0 \%$ & 0.78 \\
\hline Any grade in decreased appetite & 5 & 1.16 & $0.81-1.64$ & 0.42 & Fixed & $11 \%$ & 0.35 \\
\hline Any grade in vomiting & 4 & 1.77 & $1.11-2.84$ & 0.02 & Fixed & $27 \%$ & 0.25 \\
\hline Any grade in rash & 6 & 1.29 & $0.98-1.70$ & 0.07 & Fixed & $19 \%$ & 0.29 \\
\hline Grade 3 or higher increased ALT & 6 & 2.25 & $1.26-4.00$ & 0.006 & Fixed & $0 \%$ & 0.45 \\
\hline Grade 3 or higher increased AST & 6 & 1.89 & $0.91-3.91$ & 0.09 & Fixed & $12 \%$ & 0.34 \\
\hline Grade 3 or higher in pruritus & 6 & 0.82 & $0.22-3.10$ & 0.77 & Fixed & $0 \%$ & 0.53 \\
\hline Grade 3 or higher in diarrhea & 6 & 2.90 & $1.63-5.15$ & $<0.001$ & Fixed & $0 \%$ & 0.85 \\
\hline Grade 3 or higher in fatigue & 6 & 1.37 & $0.53-3.54$ & 0.51 & Fixed & $19 \%$ & 0.29 \\
\hline Grade 3 or higher in nausea & 5 & 2.45 & $0.71-8.51$ & 0.16 & Fixed & $0 \%$ & 0.41 \\
\hline Grade 3 or higher in vomiting & 4 & 1.63 & $0.39-6.79$ & 0.50 & Fixed & $0 \%$ & 0.51 \\
\hline Grade 3 or higher in rash & 6 & 1.69 & $0.39-7.26$ & 0.48 & Fixed & $0 \%$ & 0.51 \\
\hline
\end{tabular}

NIVO1 + IPI3, nivolumab $1 \mathrm{mg} / \mathrm{kg}$ plus ipilimumab $3 \mathrm{mg} / \mathrm{kg}$, every 3 weeks for 4 doses (induction phase), followed by nivolumab $3 \mathrm{mg} / \mathrm{kg}$, every $2 \mathrm{weeks}$ until disease progression or unacceptable toxicity incidence of TRAEs (maintenance phase); NIVO3 + IPI1, nivolumab $3 \mathrm{mg} / \mathrm{kg}$ plus ipilimumab $1 \mathrm{mg} / \mathrm{kg}$, every 3 weeks for 4 doses (induction phase), followed by nivolumab $3 \mathrm{mg} / \mathrm{kg}$, every 2 weeks until disease progression or unacceptable toxicity incidence of TRAEs (maintenance phase)

Italic indicates that the results are statistically significant, and the $P<0.05$ 
There is an increasing number of immunotherapy and molecular targeting agents being evaluated in monotherapies as well as in various combinations, but the choice of right therapy, sequence, dosage of candidate agents, immunotherapies, and treatment for patients that progress on immune checkpoint inhibitors remains a challenge.

\section{Limitation}

This meta-analysis only included four phase I clinical trials, which may reduce the credibility of the findings. In addition, this paper contains multiple tumor types, which may make the results of the study untargeted. It is necessary to point out that this paper was not able to extract hazard ratio (HR) as the effect size was insufficient, but this is the first meta-analysis to compare nivolumab plus ipilimumab with ipilimumab or nivolumab monotherapy.

\section{Conclusions}

This paper showed that the curative effect of nivolumab plus ipilimumab therapy is better than ipilimumab or nivolumab monotherapy. In the combination group, N1I3 is more effective than N3I1. Although side effects were slightly increased in group N1I3, the overall safety was reliable.

\section{Supplementary information}

Supplementary information accompanies this paper at https://doi.org/10. 1186/s12957-020-01933-5.

Additional file 1: Supplementary Table. 1. Characteristics of included clinical trials in the meta-analysis.

Additional file 2: Supplementary Figure 1. Flow chart of study selection.

\section{Acknowledgements}

Not applicable.

\section{Authors' contributions}

Conceptualization: Yingying Tang; Data curation: Jingjie Chen, Shengnan Li, and Nannan Du; Formal analysis: Jingjie Chen, Qigu Yao; Investigation: Shengnan Li, Xiaojun Fu, and Yuanmei Lou; Writing original draft: Mengru Wang, Feiyan Mao, and Danyi Mao, Parikshit Asutosh Khadaroo. The author(s) read and approved the final manuscript.

\section{Funding}

This study was funded by the Key Laboratory of Diagnosis and Treatment of Digestive System Tumors of Zhejiang Province (No. 2019E10020), Ningbo Clinical Research Center for Digestive System Tumors (No. 2019A21003), and Oncology Key Special Subject of Ningbo (2016-55).

\section{Availability of data and materials}

All data are available within the article.

\section{Ethics approval and consent to participate}

No animal or human participant was involved in this study.

\section{Consent for publication}

Not applicable.

\section{Competing interests}

The authors declare that they have no competing interests.

\section{Author details}

${ }^{1}$ Department of General Surgery, HwaMei Hospital, University of Chinese Academy of Sciences, Ningbo, Zhejiang, China. ${ }^{2}$ Ningbo Institute of Life and Health Industry, University of Chinese Academy of Sciences, Ningbo, Zhejiang, China. ${ }^{3}$ Key Laboratory of Diagnosis and Treatment of Digestive System Tumors of Zhejiang Province, Ningbo, Zhejiang, China. ${ }^{4}$ The Second Clinical Medical College, Zhejiang Chinese Medical University, Hangzhou, Zhejiang, China. ${ }^{5}$ State Key Laboratory for Diagnosis and Treatment of Infectious Diseases, the First Affiliated Hospital, College of Medicine, Zhejiang University, 79 Qingchun Rd, Hangzhou City 310003, China. ${ }^{6}$ The Third Clinical Medical College, Zhejiang Chinese Medical University, Hangzhou, Zhejiang, China. ${ }^{7}$ Medical College of Kaifeng University, Kaifeng, Henan, China. ${ }^{8}$ Basic Medical College, Zhejiang Chinese Medical University, Hangzhou, Zhejiang, China. ${ }^{9}$ Monash University School of Medicine, Nursing and Health Sciences, Melbourne, Australia. ${ }^{10}$ Department of Radiotherapy and Chemotherapy, HwaMei Hospital, University of Chinese Academy of Sciences, Northwest Street 41, Haishu District, Ningbo 315010, Zhejiang, China.

Received: 3 April 2020 Accepted: 23 June 2020

Published online: 03 July 2020

\section{References}

1. Rowshanravan $B$, Halliday N: CTLA-4: a moving target in immunotherapy. 2018, 131(1):58-67.

2. Fife BT, Bluestone JA. Control of peripheral T-cell tolerance and autoimmunity via the CTLA-4 and PD-1 pathways. Immunological reviews. 2008:224:166-82.

3. Stamper CC, Zhang Y, Tobin JF, Erbe DV, Ikemizu S, Davis SJ, Stahl ML, Seehra J, Somers WS, Mosyak L. Crystal structure of the B7-1/CTLA-4 complex that inhibits human immune responses. Nature. 2001;410(6828): 608-11.

4. Wing $K$, Onishi $Y$, Prieto-Martin $P$, Yamaguchi T, Miyara M, Fehervari Z, Nomura T, Sakaguchi S. CTLA-4 control over Foxp3+ regulatory T cell function. Science (New York, NY). 2008:322(5899):271-5.

5. Hayashi H, Nakagawa K. Combination therapy with PD-1 or PD-L1 inhibitors for cancer. Int J Clin Oncol. 2019.

6. Francisco LM, Sage PT, Sharpe AH. The PD-1 pathway in tolerance and autoimmunity. Immunological reviews. 2010;236:219-42.

7. Mahoney KM, Freeman GJ, McDermott DF. The next immune-checkpoint inhibitors: PD-1/PD-L1 blockade in melanoma. Clin Ther. 2015;37(4):764-82

8. Tay RY, Heigener D, Reck M, Califano R. Immune checkpoint blockade in small cell lung cancer. Lung cancer (Amsterdam, Netherlands). 2019;137:317.

9. McArthur HL, Page DB. Immunotherapy for the treatment of breast cancer: checkpoint blockade, cancer vaccines, and future directions in combination immunotherapy. Clinical advances in hematology \& oncology : H\&O. 2016; 14(11):922-33.

10. Liu YL, Zamarin D. Combination immune checkpoint blockade strategies to maximize immune response in gynecological cancers. Current oncology reports. 2018;20(12):94

11. Cheng H, Sun G, Chen H, Li Y, Han Z, Li Y, Zhang P, Yang L, Li Y. Trends in the treatment of advanced hepatocellular carcinoma: immune checkpoint blockade immunotherapy and related combination therapies. American journal of cancer research. 2019;9(8):1536-45.

12. Rotte A, Bhandaru M, Zhou Y, McElwee KJ. Immunotherapy of melanoma: present options and future promises. Cancer Metastasis Rev. 2015;34(1):11528

13. Rotte A, Jin JY, Lemaire V. Mechanistic overview of immune checkpoints to support the rational design of their combinations in cancer immunotherapy. Annals of oncology : official journal of the European Society for Medical Oncology. 2018;29(1):71-83.

14. Morse MA, Hochster $\mathrm{H}$, Benson A. Perspectives on treatment of metastatic colorectal cancer with immune checkpoint inhibitor therapy. Oncologist. 2019

15. El Dika I, Khalil DN, Abou-Alfa GK: Immune checkpoint inhibitors for hepatocellular carcinoma. 2019, 125(19):3312-3319.

16. Rotte A. Combination of CTLA-4 and PD-1 blockers for treatment of cancer. Journal of experimental \& clinical cancer research : CR. 2019;38(1):255. 
17. Hellmann MD, Callahan MK, Awad MM, Calvo E, Ascierto PA, Atmaca A, Rizvi NA, Hirsch FR, Selvaggi G, Szustakowski JD, et al. Tumor mutational burden and efficacy of nivolumab monotherapy and in combination with ipilimumab in small-cell lung cancer. Cancer cell. 2019;35(2):329.

18. Hellmann MD, Paz-Ares L, Bernabe Caro R, Zurawski B, Kim SW, Carcereny Costa E, Park K, Alexandru A, Lupinacci L, de la Mora JE, et al. Nivolumab plus ipilimumab in advanced non-small-cell lung cancer. New England J Med. 2019.

19. Antonia SJ, Lopez-Martin JA, Bendell J, Ott PA, Taylor M, Eder JP, Jager D, Pietanza MC, Le DT, de Braud F, et al. Nivolumab alone and nivolumab plus ipilimumab in recurrent small-cell lung cancer (CheckMate 032): a multicentre, open-label, phase 1/2 trial. The Lancet Oncology. 2016;17(7): 883-95.

20. D'Angelo SP, Mahoney MR, Van Tine BA, Atkins J, Milhem MM, Jahagirdar BN, Antonescu CR, Horvath E, Tap WD, Schwartz GK, et al. Nivolumab with or without ipilimumab treatment for metastatic sarcoma (Alliance A091401): two open-label, non-comparative, randomised, phase 2 trials. Lancet Oncol. 2018;19(3):416-26.

21. Hammers HJ, Plimack ER, Infante JR, Rini Bl, McDermott DF, Lewis LD, Voss MH, Sharma P, Pal SK, Razak ARA, et al. Safety and efficacy of nivolumab in combination with ipilimumab in metastatic renal cell carcinoma: the CheckMate 016 study. J Clin Oncol. 2017;35(34):3851-8.

22. Hodi FS, Chiarion-Sileni V, Gonzalez R, Grob JJ, Rutkowski P, Cowey CL, Lao CD, Schadendorf D, Wagstaff J, Dummer R, et al. Nivolumab plus ipilimumab or nivolumab alone versus ipilimumab alone in advanced melanoma (CheckMate 067): 4-year outcomes of a multicentre, randomised, phase 3 trial. The Lancet Oncology. 2018;19(11):1480-92.

23. Janjigian YY, Bendell J, Calvo E, Kim JW, Ascierto PA, Sharma P, Ott PA Peltola K, Jaeger D, Evans J, et al. CheckMate-032 study: efficacy and safety of nivolumab and nivolumab plus ipilimumab in patients with metastatic esophagogastric cancer. Journal of clinical oncology : official journal of the American Society of Clinical Oncology. 2018;36(28):2836-44.

24. Lebbe C, Meyer N, Mortier L, Marquez-Rodas I, Robert C, Rutkowski P, Menzies AM, Eigentler T, Ascierto PA, Smylie M, et al. Evaluation of two dosing regimens for nivolumab in combination with ipilimumab in patients with advanced melanoma: results from the phase IIIb/IV CheckMate 511 trial. Journal of clinical oncology : official journal of the American Society of Clinical Oncology. 2019;37(11):867-75

25. Long GV, Atkinson V, Lo S, Sandhu S, Guminski AD, Brown MP, Wilmott JS, Edwards J, Gonzalez M, Scolyer RA, et al. Combination nivolumab and ipilimumab or nivolumab alone in melanoma brain metastases: a multicentre randomised phase 2 study. The Lancet Oncology. 2018;19(5):672-81.

26. Omuro A, Vlahovic G, Lim M, Sahebjam S, Baehring J, Cloughesy T, Voloschin A, Ramkissoon SH, Ligon KL, Latek R, et al. Nivolumab with or without ipilimumab in patients with recurrent glioblastoma: results from exploratory phase I cohorts of CheckMate 143. Neuro-oncology. 2018;20(5): 674-86.

27. Postow MA, Chesney J, Pavlick AC, Robert C, Grossmann K, McDermott D, Linette GP, Meyer N, Giguere JK, Agarwala SS, et al. Nivolumab and ipilimumab versus ipilimumab in untreated melanoma. The New England journal of medicine. 2015;372(21):2006-17

28. Scherpereel A, Mazieres J, Greillier L, Lantuejoul S, Do P, Bylicki O, Monnet I, Corre R, Audigier-Valette C, Locatelli-Sanchez M, et al. Nivolumab or nivolumab plus ipilimumab in patients with relapsed malignant pleural mesothelioma (IFCT-1501 MAPS2): a multicentre, open-label, randomised, non-comparative, phase 2 trial. The Lancet Oncology. 2019;20(2):239-53.

29. Sharma P, Siefker-Radtke A, de Braud F, Basso U, Calvo E, Bono P, Morse MA, Ascierto PA, Lopez-Martin J, Brossart P, et al. Nivolumab alone and with ipilimumab in previously treated metastatic urothelial carcinoma: CheckMate 032 nivolumab 1 mg/kg plus ipilimumab 3 mg/kg expansion cohort results. Journal of clinical oncology : official journal of the American Society of Clinical Oncology. 2019;37(19):1608-16.

30. Ghysen K, Vansteenkiste J. Immunotherapy in patients with early stage resectable nonsmall cell lung cancer. Current opinion in oncology. 2019; 31(1):13-7.

31. Wrona A. Role of immunotherapy in stage III nonsmall cell lung cancer. Current opinion in oncology. 2019;31(1):18-23.

32. Lasinska I, Kolenda T, Teresiak A, Lamperska KM, Galus L, Mackiewicz J. Immunotherapy in patients with recurrent and metastatic squamous cell carcinoma of the head and neck. Anti-cancer agents in medicinal chemistry. 2019:19(3):290-303.
33. Tang Y, Li G, Wu S, Tang L, Zhang N, Liu J, Zhang S, Yao L. Programmed death ligand 1 expression in esophageal cancer following definitive chemoradiotherapy: prognostic significance and association with inflammatory biomarkers. Oncology letters. 2018;15(4):4988-96.

34. Blumenthal GM, Bunn PA Jr, Chaft JE, McCoach CE, Perez EA, Scagliotti GV, Carbone DP, Aerts $\mathrm{H}$, Aisner DL, Bergh J, et al. Current status and future perspectives on neoadjuvant therapy in lung cancer. J Thoracic Oncol. 2018; 13(12):1818-31.

35. Tarhini A, Kudchadkar RR. Predictive and on-treatment monitoring biomarkers in advanced melanoma: moving toward personalized medicine. Cancer treatment reviews. 2018;71:8-18.

36. Schadendorf D, Ascierto PA, Haanen J, Espinosa E, Demidov L, Garbe C, Guida M, Lorigan P, Chiarion-Sileni V, Gogas H, et al. Safety and efficacy of nivolumab in challenging subgroups with advanced melanoma who progressed on or after ipilimumab treatment: a single-arm, open-label, phase II study (CheckMate 172). Eur J Cancer (Oxford, England : 1990). 2019; 121:144-53.

37. Nomura M, Otsuka A, Kondo T, Nagai H, Nonomura Y, Kaku Y, Matsumoto S, Muto M. Efficacy and safety of retreatment with nivolumab in metastatic melanoma patients previously treated with nivolumab. Cancer chemotherapy and pharmacology. 2017:80(5):999-1004.

38. Cetin B, Kosar A. Game of thrones: immunotherapy versus molecular targeted therapy in renal cell cancer scenarios. International urology and nephrology. 2019

39. Ciardiello D, Vitiello PP, Cardone C, Martini G, Troiani T, Martinelli E, Ciardiello F. Immunotherapy of colorectal cancer: challenges for therapeutic efficacy. Cancer treatment reviews. 2019;76:22-32.

40. Cope S, Keeping ST, Goldgrub R, Ayers D, Jansen JP, Penrod JR, Korytowsky $B$, Juarez-Garcia A, Yuan Y. Indirect comparison of nivolumab +/ipilimumab (CheckMate 032) versus other treatments for recurrent small-cell lung cancer. Journal of comparative effectiveness research. 2019;8(10):73351.

41. Ready N, Hellmann MD, Awad MM, Otterson GA, Gutierrez M, Gainor JF, Borghaei $H$, Jolivet J, Horn L, Mates M, et al. First-line nivolumab plus ipilimumab in advanced non-small-cell lung cancer (CheckMate 568): outcomes by programmed death ligand 1 and tumor mutational burden as biomarkers. Journal of clinical oncology : official journal of the American Society of Clinical Oncology. 2019;37(12):992-1000.

42. Shao N, Wan F, Zhu Y, Ye D. Conditional survival in patients with advanced renal cell carcinoma treated with nivolumab. Medical science monitor : international medical journal of experimental and clinical research. 2019;25: 6518-22.

43. Larkin J, Chiarion-Sileni V, Gonzalez R, Grob JJ, Rutkowski P, Lao CD, Cowey $C L$, Schadendorf D, Wagstaff J, Dummer R et al: Five-year survival with combined nivolumab and ipilimumab in advanced melanoma. 2019.

44. Valpione S, Campana LG. Immunotherapy for advanced melanoma: future directions. Immunotherapy. 2016;8(2):199-209.

45. Muto Y. Kitano S: [Clinical significance of ipilimumab and the combination therapy as immune checkpoint inhibitor]. Gan to kagaku ryoho Cancer Chemother. 2019;46(6):1011-5.

46. Freeman M, Betts KA, Jiang S, Du EX, Gupte-Singh K, Lu Y, Rao S, Shoushtari AN: Indirect treatment comparison of nivolumab versus observation or ipilimumab as adjuvant therapy in resected melanoma using pooled clinical trial data. Advances in therapy 2019.

47. Motzer RJ, Rini BI, McDermott DF, Aren Frontera O, Hammers HJ, Carducci MA, Salman P, Escudier B, Beuselinck B, Amin A, et al. Nivolumab plus ipilimumab versus sunitinib in first-line treatment for advanced renal cell carcinoma: extended follow-up of efficacy and safety results from a randomised, controlled, phase 3 trial. Lancet Oncol. 2019;20(10):1370-85.

48. Weber JS, D'Angelo SP, Minor D, Hodi FS, Gutzmer R, Neyns B, Hoeller C, Khushalani NI, Miller WH Jr, Lao CD, et al. Nivolumab versus chemotherapy in patients with advanced melanoma who progressed after anti-CTLA-4 treatment (CheckMate 037): a randomised, controlled, open-label, phase 3 trial. Lancet Oncol. 2015;16(4):375-84.

49. McDermott DF, Shah R, Gupte-Singh K, Sabater J, Luo L, Botteman M, Rao S, Regan MM, Atkins M. Quality-adjusted survival of nivolumab plus ipilimumab or nivolumab alone versus ipilimumab alone among treatmentnaive patients with advanced melanoma: a quality-adjusted time without symptoms or toxicity (Q-TWiST) analysis. Quality Life Res. 2019;28(1):109-19.

50. Francisco LM, Salinas VH, Brown KE, Vanguri VK, Freeman GJ, Kuchroo VK, Sharpe AH. PD-L1 regulates the development, maintenance, and function of 
induced regulatory T cells. The Journal of experimental medicine. 2009; 206(13):3015-29.

51. Motzer RJ, Rini BI, McDermott DF, Redman BG, Kuzel TM, Harrison MR, Vaishampayan UN, Drabkin HA, George S, Logan TF, et al. Nivolumab for metastatic renal cell carcinoma: results of a randomized phase II trial. J Clin Oncol. 2015;33(13):1430-7.

52. Neoadjuvant PD-1 Blockade in resectable lung cancer; nivolumab and ipilimumab in advanced melanoma; overall survival with combined nivolumab and ipilimumab in advanced melanoma; prolonged survival in stage III melanoma with ipilimumab adjuvant therapy; combined nivolumab and ipilimumab or monotherapy in untreated melanoma; combined nivolumab and ipilimumab or monotherapy in untreated melanoma; nivolumab and ipilimumab versus ipilimumab in untreated melanoma; rapid eradication of a bulky melanoma mass with one dose of immunotherapy; genetic basis for clinical response to CTLA-4 blockade; genetic basis for clinical response to CTLA-4 blockade in melanoma; nivolumab plus ipilimumab in advanced melanoma; safety and tumor responses with lambrolizumab (anti-PD-1) in melanoma; hepatotoxicity with combination of vemurafenib and ipilimumab. The New England journal of medicine 2018, 379(22):2185.

53. Ott PA, Hodi FS, Robert C. CTLA-4 and PD-1/PD-L1 blockade: new immunotherapeutic modalities with durable clinical benefit in melanoma patients. Clinical cancer research : an official journal of the American Association for Cancer Research. 2013;19(19):5300-9.

54. Buchbinder El, Desai A. CTLA-4 and PD-1 pathways: similarities, differences, and implications of their inhibition. American journal of clinical oncology. 2016;39(1):98-106

55. Ghiringhelli F, Fumet JD. Is there a place for immunotherapy for metastatic microsatellite stable colorectal cancer? Front Immunol. 2019;10:1816.

56. Ready NE, Ott PA, Hellmann MD, Zugazagoitia J, Hann CL, de Braud F, Antonia SJ, Ascierto PA, Moreno V, Atmaca A, et al. Nivolumab monotherapy and nivolumab plus ipilimumab in recurrent small cell lung cancer: results from the CheckMate 032 randomized cohort. Journal of thoracic oncology : official publication of the International Association for the Study of Lung Cancer. 2020;15(3):426-35.

57. Kreft S, Gesierich A, Eigentler T, Franklin C, Valpione S, Ugurel S, Utikal J, Haferkamp S, Blank C, Larkin J, et al. Efficacy of PD-1-based immunotherapy after radiologic progression on targeted therapy in stage IV melanoma. Eur J Cancer (Oxford, England : 1990). 2019;116:207-15.

58. Fujimura T, Kambayashi Y, Sato Y, Tanita K, Amagai R, Hashimoto A, Hidaka T, Aiba S. Successful treatment of unresectable advanced melanoma by administration of nivolumab with ipilimumab before primary tumor resection. Front Med. 2019;6:140.

59. Glutsch V, Gran F, Weber J, Gesierich A, Goebeler M, Schilling B: Response to combined ipilimumab and nivolumab after development of a nephrotic syndrome related to PD-1 monotherapy. 2019, 7(1):181.

60. Wen X, Wang Y, Ding Y, Li D, Li J, Guo Y, Peng R, Zhao J, Zhang X, Zhang $X S$. Safety of immune checkpoint inhibitors in Chinese patients with melanoma. Melanoma research. 2016;26(3):284-9.

61. Bajwa R, Cheema A, Khan T, Amirpour A, Paul A, Chaughtai S, Patel S, Patel T, Bramson J, Gupta V, et al. Adverse effects of immune checkpoint inhibitors (programmed death-1 inhibitors and cytotoxic T-lymphocyteassociated protein-4 inhibitors): results of a retrospective study. Journal of clinical medicine research. 2019;11(4):225-36.

62. Pollack MH, Betof A, Dearden H, Rapazzo K, Valentine I, Brohl AS, Ancell KK Long GV, Menzies AM, Eroglu Z, et al. Safety of resuming anti-PD-1 in patients with immune-related adverse events (irAEs) during combined antiCTLA-4 and anti-PD1 in metastatic melanoma. Annals of oncology : official journal of the European Society for Medical Oncology. 2018;29(1):250-5.

\section{Publisher's Note}

Springer Nature remains neutral with regard to jurisdictional claims in published maps and institutional affiliations.

Ready to submit your research? Choose BMC and benefit from:

- fast, convenient online submission

- thorough peer review by experienced researchers in your field

- rapid publication on acceptance

- support for research data, including large and complex data types

- gold Open Access which fosters wider collaboration and increased citations

- maximum visibility for your research: over $100 \mathrm{M}$ website views per year

At BMC, research is always in progress.

Learn more biomedcentral.com/submissions 